\title{
Effect of Perioperative Nursing on the Prevention of Lower Extremity Deep Vein Thrombosis in Patients Undergoing Gynecological Pelvic Surgery
}

\author{
Lirong Mo, Xiaojuan Liao*, Huijuan Cai \\ Gynecology Department, The First Affiliated Hospital of Jinan University, Guangzhou, China \\ Email address: \\ 804893466@qq.com (Lirong Mo),L8808104@126.com (Xiaojuan Liao), 15975482120@163.com (Huijuan Cai) \\ ${ }^{*}$ Corresponding author
}

\section{To cite this article:}

Lirong Mo, Xiaojuan Liao, Huijuan Cai. Effect of Perioperative Nursing on the Prevention of Lower Extremity Deep Vein Thrombosis in Patients Undergoing Gynecological Pelvic Surgery. American Journal of Nursing Science. Vol. 9, No. 6, 2020, pp. 455-458.

doi: $10.11648 /$ j.ajns.20200906.23

Received: December 5, 2020; Accepted: December 17, 2020; Published: December 22, 2020

\begin{abstract}
Background: Deep venous thrombosis (DVT) is a common complication of gynecological pelvic surgery. DVT focuses on prevention. How to make a detailed perioperative nursing plan and organize its implementation of the measures is a great significance for the prevention of DVT in patients undergoing gynecological pelvic surgery. Objective: To investigate the effect of perioperative nursing intervention measures on the prevention of lower extremity deep vein thrombosis (DVT) in patients undergoing pelvic surgery in gynecology. Methods: Set up a project improvement activity group, analyzation the reason of gynecological pelvic surgery patients with DVT, and development a detailed plan of perioperative nursing intervention measures. Organizing the implementation of "one-on-one" nursing intervention, the problems in the nursing process were collected and formulated solutions, inspection gaps in care, made up the nursing error and improved nursing content. It was used to improve the quality of preventive measures and promote their implementation by "plan-do-check-action" (PDCA) cycle. Results: The perioperative preventive measures were continuously improved with PDCA cycle. The incidence of DVT in patients with pelvic surgery in our gynecological ward decreased from $6.96 \%$ to $2.08 \%$. The anus exhausting time, resumption of eating time, defecation time and first leaving bed time of perioperative nursing group were shorter than before of the project improvement activities. The hospitalization time of patients was also significantly shortened. The differences were statistically significant $(P<0.05)$. Through the follow-up survey, patients' satisfaction with nursing increased from $82.6 \%$ to $95.0 \%$. Conclusion: Through the development of detailed perioperative nursing plan and promotion the implementation of the measures with PDCA cycle continuously improved for the prevention of DVT in patients undergoing gynecological pelvic surgery, the incidence of postoperative lower extremity DVT could be effectively reduced and also promote the rehabilitation of postoperative. It was significantly shortened in the hospitalization time of patients, and improved the satisfaction of patients in perioperative nursing.
\end{abstract}

Keywords: Perioperative Nursing, Gynecological Pelvic Surgery, Lower Extremity Deep Vein Thrombosis, Project Improvement

\section{Introduction}

Deep venous thrombosis (DVT) is a venous reflux disorder caused by abnormal blood coagulation in the deep vein [1]. DVT usually occurs in the lower limbs, which the thrombus shedding can cause fatal pulmonary embolism [2]. The incidence of DVT varies from surgical type [3]. The investigation shows that the incidence of lower extremity DVT in gynecologic pelvic surgery patients is $9.2 \%$ to $15.6 \%$ [4], which is due to body position, intraoperative vascular injury, slow blood flow, high blood coagulation, etc [5]. DVT focuses on prevention [6]. It has a great significance to prevent the occurrence of DVT in patients with perioperative nursing intervention [7]. In this study, we established a special project improvement activity group, analyzed the reason of gynecological pelvic surgery patients with DVT, and 
developed a detailed plan of perioperative nursing intervention measures. Through the continuous improvement of "plan-do-check-action" (PDCA) cycle [8] for the prevention of DVT and promoting the implementation of the measures, satisfactory results have been obtained, as reported below.

\section{Materials and Methods}

\subsection{General Information}

The subjects were patients who received pelvic surgery in our gynecology department from October 2019 to October 2020. Inclusive criteria: (1) patients receiving pelvic surgery in gynecology department; (2) hospitalization time > 2 days; (3) age $\geq 18$ years old; (4) informed consent. Exclusion criteria: patients with DVT of lower extremity. According to the time sequence, the patients who received routine nursing from October 2019 to March 2020 were selected as the control group. After April 2020, a project improvement group was set up to analyze the causes of DVT in patients undergoing gynecological pelvic surgery, search for the best evidence of nursing to prevent DVT, formulate a detailed perioperative nursing plan [9]. It was used to improve the quality of preventive measures and promote the implementation of the measures by PDCA cycle. Patients who received perioperative nursing with PDCA cycle from May 2020 to October 2019 were selected as the observation group. Among them, 230 patients in the control group, aged 35 74 (55.2 \pm 10.5$)$ years old, 240 cases in the observation group, aged 32 73 (54.5 \pm 10.6) years. The types of surgery include: ovarian surgery in 66 cases, 69 cases; tubal surgery in 48 cases, 51 cases; uterine surgery in 84 cases, 88 cases; pelvic surgery in 32 cases, 32 cases. The general data of the two groups were comparable $(P>0.05)$.

\subsection{Methods}

\subsubsection{Set up a Project Improvement Activity Group}

Set up a project improvement activity group composed of group leader, counselor and team members, who formulated the activity schedule, analyzed the causes of DVT in patients with gynecological pelvic surgery by brainstorming, fishbone diagram, current situation investigation, cause analysis, and cause demonstration, searched for the best evidence of nursing to prevent DVT, and formulated detailed perioperative nursing care plan and organized the implementation.

\subsubsection{Perioperative Nursing Intervention Measures}

(1) Risk assessment: After admission, the patients were given preoperative Wells score of lower extremity DVT, and their past medical history were inquired in detail. For patients with history of thromboembolism, hypertension, diabetes, hyperlipidemia and other diseases, as well as those with score $\geq 1$, key prevention and treatment should be carried out [10]. In the case of no contraindications, low molecular weight heparin could be given subcutaneous injection of anticoagulant therapy to prevent the formation of postoperative lower extremity DVT [11]. Strengthen health education to improve patients' awareness of DVT.
(2) Psychological intervention: Before operation, we should fully communication with the patients, trying to reduce the fear and anxiety of the operation, which to dredge the bad psychological mood, enhance the patients' confidence in surgery, so as to enhance the compliance of treatment and nursing.

(3) Strengthen the observation of lower limbs blood supply: Measured the circumference of thigh and leg before operation, observed and recorded the circulation of extremities, including: whether the skin temperature of legs was increased, whether there was swelling, numbness, whether the color was changed, and whether the pulse of dorsal artery of foot was weakened.

(4) Intraoperative nursing: During the operation, the patient should keep a proper and comfortable position. High appropriate cushion should be placed on the lower leg and popliteal fossa in order to avoid blood circulation disorder caused by long-time compression of venous plexus. At the same time, elastic stockings were worn to promote venous blood return.

(5) Postoperative nursing: The patient returned to the ward after surgery, during the period of absolute bed rest, the nursing bed was shaken to the high position of foot, so that the lower limbs were raised by $20^{\circ}$ to $30^{\circ}$. Patients were required to continue to wear elastic stockings to increase venous reflux and reduce the risk of thrombosis. It was forbidden to squeeze the lower limbs after operation, while could massage the lower limbs 4 5 times a day for about 10 minutes each time, and kept the extremities warm [12]. The vital signs and limb circulation were recorded every day after the operation, and the circumference of thigh and leg were measured. If the difference between the measurements results before operation, or the results of left and right legs were too large, we should be alert to the possibility of venous thrombosis, and there were need to improve the color Doppler ultrasound examination of lower extremity veins as soon as possible [13].

(6) Guiding rehabilitation exercise: It was important to take rehabilitation exercise in time after operation to prevent venous thrombosis. If the patient's condition permits, they should be encouraged to carry out activities as soon as possible to promote the recovery of lower limbs function. It could increase the femoral vein blood flow velocity with early lower limb exercise, especially knee joint extension and flexion, ankle active and passive movement [14]. The family members of the patient were urged to help them turn over, change positions, move the joints of the lower limbs and massage the muscles of the extremities every $2 \sim 3$ hours, and also encouraged patients to do flexion and extension of toes, ankle joint and other activities in bed.

\subsubsection{Implementation and Improvement of Perioperative Nursing Intervention Measures}

According to the perioperative nursing plan, the nursing staffs in the group were arranged to implement the intervention 
measures with "one-to-one" nursing for patients. The problems existing in the nursing process were collected, and the errors in the nursing process were recorded, which were discussed and set a solution plan to make up for the nursing loopholes or errors. The existing problems were addressed, and the contents of nursing were constantly improved to ensure the smooth implementation of perioperative nursing plan for patients' rehabilitation. The quality of nursing was improved by PDCA continuous circulation.

\subsection{Observation Index}

The incidence of lower extremity DVT was compared between the two groups before and after the project improvement activity. They were compared between the two groups in the anus exhausting time, resumption of eating time, defecation time, first leaving bed time and hospitalization time, etc. The patients' satisfaction of nursing was also investigated with questionnaire.

\subsection{Statistical Methods}

SPSS21.0 statistical software was used to statistical analysis. Measurement data were expressed as mean \pm standard deviation $(\bar{x} \pm s)$, and comparison between groups was performed by $t$ test. Statistical data were expressed in terms of frequency and percentage (\%), when chi-square test was used. The difference was statistically significant with $P<0.05$.

\section{Results}

\subsection{Incidence of Lower Extremity DVT}

Through continuously improved in perioperative nursing measures with PDCA cycle, the incidence of lower extremity DVT in patients with gynecological pelvic surgery in the ward decreased from $6.96 \%$ in 16 cases $(16 / 230)$ to $2.08 \%$ in 5 cases $(5 / 240)$. The difference was statistically significant $(P<$ $0.05)$, as shown in Table 1.

Table 1. Comparison of the incidence of lower extremity DVT between the two groups (Cases).

\begin{tabular}{llll}
\hline Projects & Cases & DVT Cases & Incidence \\
\hline Routine nursing group & 230 & 16 & $6.96 \%$ \\
Perioperative nursing group & 240 & 5 & $2.08 \%$ \\
$\chi^{2}$ & & & 6.534 \\
$P$ & & & 0.011 \\
\hline
\end{tabular}

\subsection{Postoperative Recovery and Hospitalization Time}

The anus exhausting time, resumption of eating time, defecation time, first leaving bed time and hospitalization time of perioperative nursing group were shorter than those of routine nursing group, with a statistically significant difference $(P<0.05)$, as shown in Table 2. Through the follow-up survey, patients' satisfaction with nursing increased from $82.6 \%$ to $95.0 \%$. The difference was also statistically significant $(P=0.000)$.

Table 2. Comparison of the postoperative recovery and hospitalization time between the two groups $(\bar{x} \pm s)$.

\begin{tabular}{llll}
\hline Projects & Routine nursing group (n=230) & Perioperative nursing group (n=240) & $\boldsymbol{t}$ \\
\hline Anus exhausting time (hours) & $10.4 \pm 2.2$ & $9.0 \pm 1.5$ & 8.123 \\
Resumption of eating time (hours) & $10.4 \pm 2.0$ & $9.1 \pm 1.3$ & 0.000 \\
Defecation time (hours) & $37.9 \pm 6.6$ & $33.7 \pm 5.8$ & 0.000 \\
First time out of bed time (hours) & $30.7 \pm 6.6$ & $26.4 \pm 6.1$ & 7.502 \\
Hospitalization time (days) & $9.9 \pm 3.9$ & $8.5 \pm 3.0$ & 7.271 \\
\hline
\end{tabular}

\section{Discussion}

Gynecological pelvic surgery is one of the common clinical operations in modern medicine, which is often used to treat female pelvic diseases with ideal effect, such as ectopic pregnancy, ovarian cyst, uterine fibroids and pelvic tumors, etc. But after pelvic surgery, there will be some complications, among which lower extremity DVT is a common complication of pelvic surgery [15]. Due to surgical reasons, the patient has to stay in bed for a long time. In addition, the pain will brake the limbs and reduce the movement. The blood flow of the lower limbs is slow and the blood is in a hypercoagulable state. The investigation shows that the incidence of DVT in patients with gynecological pelvic surgery is as high as $9.2 \% \sim 15.6 \%$ [16]. DVT focuses on prevention. It is a great significance for the prevention of DVT with nursing intervention during perioperative period.

Routine nursing is the most widely used nursing mode in clinical nursing work, but it is only a simple intervention on the patient's condition, lifestyle, treatment and other aspects, lacking specificity and comprehensiveness. While perioperative nursing has a more in-depth communication with patients on the basis of routine nursing [17], so that patients can have a detailed understanding of their own condition, surgery, complications, etc. This helps patients to overcome the fear and anxiety of surgery, and at the same time, early intervention measures will be taken for high-risk groups with DVT. In this study, through establishment of a project improvement activity group and development of activity schedule, the causes of DVT in patients with gynecological pelvic surgery were analyzed by brainstorming, fishbone diagram, current situation investigation, cause analysis, and cause demonstration. The best evidence of nursing were searched to prevent DVT of lower limbs, while the perioperative nursing intervention measures were formulate based on the evidence-based [18]. According to the perioperative nursing plan, the nursing staffs in the group were arranged to implement the intervention measures with one-to-one nursing for patients. The perioperative nursing intervention measures were implemented from the aspects of risk assessment, psychological intervention, observation of lower limbs blood supply, intraoperative and postoperative nursing, rehabilitation exercise, etc. The existing problems in the nursing process were collected, and the errors in the nursing process were recorded, which were discussed and set 
a solution plan to make up for the nursing loopholes or errors. The nursing content was constantly improvement by PDCA cycle, which ensured the perioperative nursing plan smooth implementation to promote rehabilitation of patients.

Through continuously improved in perioperative nursing measures with PDCA cycle, the incidence of lower extremity DVT in patients with gynecological pelvic surgery in our ward decreased from $6.96 \%$ to $2.08 \%$. The anus exhausting time, resumption of eating time, defecation time, first leaving bed time and hospitalization time of perioperative nursing group were shorter than those of routine nursing group, with a statistically significant difference $(P<0.05)$. Through the follow-up survey, patients' satisfaction with perioperative nursing was significantly higher than that before of the activity. Continuous improvement of perioperative nursing measures could promote the rehabilitation of patients with gynecological pelvic surgery.

\section{Conclusion}

Development of detailed perioperative nursing plan and promotion the implementation of the measures with PDCA cycle continuously improved could be effectively reduced the incidence of postoperative lower extremity in patients undergoing gynecological pelvic surgery. Those continuous improvements of perioperative nursing measures could promote the rehabilitation of patients, shorten the hospitalization time significantly, and the patients' satisfaction was also improved. Perioperative nursing was worthy of clinical application. In prevent the lower extremity DVT, while the nursing measures required continuous improvement and needed to organize the implementation of "one-on-one" nursing intervention.

\section{References}

[1] Robert-Ebadi H, Righini M. Management of distal deep vein thrombosis. Thromb Res. 2017; 149: 48-55.

[2] Di Nisio M, van Es N, Büller HR. Deep vein thrombosis and pulmonary embolism. Lancet. 2016; 388 (10063): 3060-3073.

[3] Graul A, Latif N, Zhang X, et al. Incidence of Venous Thromboembolism by Type of Gynecologic Malignancy and Surgical Modality in the National Surgical Quality Improvement Program. Int J Gynecol Cancer. 2017; 27 (3): 581-587.

[4] Trugilho IA, Renni MJP, Medeiros GC, Thuler LCS, Bergmann A. Incidence and factors associated with venous thromboembolism in women with gynecologic cancer [J]. Thromb Res. 2020; 185: 49-54.

[5] McLendon K, Goyal A, Bansal P, Attia M. Deep Venous Thrombosis Risk Factors. In: StatPearls. Treasure Island (FL): StatPearls Publishing; April 29, 2020.

[6] Badireddy M, Mudipalli VR. Deep Venous Thrombosis Prophylaxis. In: StatPearls. Treasure Island (FL): StatPearls Publishing; September 10, 2020.

[7] Qing C, Yuanyuan J, Deling W, et al. Nursing Care of Gynecological Pelvic Surgery for Prevention of Deep Venous Thrombosis of Lower Extremity [J]. Biped and Health, 2018,
27 (24): $15-16$.

[8] Isniah S, Purba H H, Debora F. Plan do check action (PDCA) method: literature review and research issues [J]. Jurnal Sistem dan Manajemen Industri, 2020, 4 (1): 72-81.

[9] Rausa E, Kelly ME, Asti E, et al. Extended versus conventional thromboprophylaxis after major abdominal and pelvic surgery: Systematic review and meta-analysis of randomized clinical trials. Surgery. 2018; 164 (6): 1234-1240.

[10] Paik B, Joh JH, Park HC. Anatomic and clinical risk factors for pulmonary embolism in patients with deep venous thrombosis of the lower extremity. Ann Surg Treat Res. 2017; 92 (5): 365-369.

[11] Felder S, Rasmussen MS, King R, et al. Prolonged thromboprophylaxis with low molecular weight heparin for abdominal or pelvic surgery. Cochrane Database Syst Rev. 2018; 11 (11): CD004318. Published 2018 Nov 27.

[12] Yu R, Nansubuga F, Yang J, et al. Efficiency and safety evaluation of prophylaxes for venous thrombosis after gynecological surgery. Medicine (Baltimore). 2020; 99 (25): e20928.

[13] Needleman L, Cronan JJ, Lilly MP, et al. Ultrasound for Lower Extremity Deep Venous Thrombosis: Multidisciplinary Recommendations From the Society of Radiologists in Ultrasound Consensus Conference. Circulation. 2018; 137 (14): 1505-1515.

[14] Guo M, Lu L, Sun Y, Li L, Wu M, Lang J. Comprehensive functional exercises with patient education for the prevention of venous thrombosis after major gynecologic surgery: A randomized controlled study. Thromb Res. 2019; 178: 69-74.

[15] Hattab Y, Küng S, Fasanya A, Ma K, Singh AC, DuMont T. Deep Venous Thrombosis of the Upper and Lower Extremity. Crit Care Nurs Q. 2017; 40 (3): 230-236.

[16] Cohen A, Lim CS, Davies AH. Venous Thromboembolism in Gynecological Malignancy. Int J Gynecol Cancer. 2017; 27 (9): 1970-1978.

[17] Kuster Uyeda MGB, Batista Castello Girão MJ, Carbone ÉDSM, Machado Fonseca MC, Takaki MR, Ferreira Sartori MG. Fast-track protocol for perioperative care in gynecological surgery: Cross-sectional study. Taiwan J Obstet Gynecol. 2019; 58 (3): 359-363.

[18] Mahdi H, Aljebori Q, Lockart D, Moulton L. Risk of Venous Thromboembolism After Laparoscopic Surgery for Gynecologic Malignancy. J Minim Invasive Gynecol. 2016; 23 (7): 1057-1062.

\section{Biography}

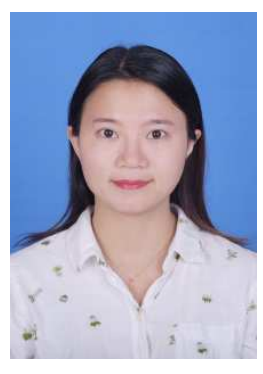

Lirong Mo, Female, Bachelor, Nurse practitioner, Dedicated to gynaecological clinical nursing and gynecological ward management. 